\title{
MAXIMAL SUBSETS OF PAIRWISE NONCOMMUTING ELEMENTS OF THREE-DIMENSIONAL GENERAL LINEAR GROUPS
}

\author{
AZIZOLLAH AZAD ${ }^{\bowtie}$ and CHERYL E. PRAEGER
}

(Received 18 August 2008)

\begin{abstract}
Let $G$ be a group. A subset $N$ of $G$ is a set of pairwise noncommuting elements if $x y \neq y x$ for any two distinct elements $x$ and $y$ in $N$. If $|N| \geq|M|$ for any other set of pairwise noncommuting elements $M$ in $G$, then $N$ is said to be a maximal subset of pairwise noncommuting elements. In this paper we determine the cardinality of a maximal subset of pairwise noncommuting elements in a threedimensional general linear group. Moreover, we show how to modify a given maximal subset of pairwise noncommuting elements into another maximal subset of pairwise noncommuting elements that contains a given 'generating element' from each maximal torus.
\end{abstract}

2000 Mathematics subject classification: primary 20D60.

Keywords and phrases: singer cycle subgroup, unipotent element, general linear group.

\section{Introduction}

Let $G$ be a nonabelian group and $Z(G)$ be its centre. We call a subset $N$ of $G$ a set of pairwise noncommuting elements if $x y \neq y x$ for any distinct elements $x, y$ in $N$. If $|N| \geq|M|$ for any other subset of pairwise noncommuting elements $M$ in $G$, then $N$ is said to be a maximal subset of pairwise noncommuting elements. The cardinality of such a subset is denoted by $\omega(G)$. By a famous result of Neumann [9] in answer to a question posed by P. Erdős, the finiteness of $\omega(G)$ in $G$ is equivalent to the finiteness of the factor group $G / Z(G)$. Mason [8] has shown that any finite group $G$ can be covered by at most $[|G| / 2]+1$ abelian subgroups, so we also have $\omega(G) \leq[|G| / 2]+1$. Moreover, $\omega(G)$ is also related to the index of the centre of $G$ : as Pyber [10] has shown, there is some constant $c$ such that $|G: Z(G)| \leq c^{\omega(G)}$. For a prime number $p$, a finite $p$-group $G$ is called extra-special if the centre, the Frattini subgroup and the derived subgroup of $G$ all coincide and are cyclic of order $p$. The cardinalities of maximal subsets of pairwise noncommuting elements of extra-special $p$-groups are important as they provide combinatorial information which can be used to calculate their cohomology lengths. (The cohomology length of a nonelementary

(C) 2009 Australian Mathematical Society 0004-9727/2009 \$16.00 
abelian $p$-group is a cohomology invariant defined as a result of a theorem of Serre [11].) Chin [4] has obtained upper and lower bounds for $\omega(G)$ for extra-special $p$ groups $G$, for odd prime numbers $p$. For $p=2$, it has been shown by Isaacs (see [2, p. 40]) that $\omega(G)=2 n+1$ for any extra-special group of order $2^{2 n+1}$. Also, in [1, Lemma 4.4], it was proved that $\omega(G L(2, q))=q^{2}+q+1$. In this paper we determine $\omega(G L(3, q))$.

TheOREM 1.1.

$$
\omega(G L(3, q))= \begin{cases}q^{6}+q^{5}+3 q^{4}+3 q^{3}+q^{2}-q-1 & \text { if } q \geq 4 \\ 1067 & \text { if } q=3 \\ 57 & \text { if } q=2\end{cases}
$$

We believe that a similar result may hold for higher dimensions.

Conjecture 1.2. Let $G=G L(n, q)$, where $q=p^{k} \geq 4$ and $q \geq n+1$. Then

$$
\omega(G) \geq q^{2\left(\begin{array}{c}
n \\
2
\end{array}\right)}+\frac{|G|}{q(q-1)^{n}}+\frac{|G|}{q^{\left(\begin{array}{c}
n \\
2
\end{array}\right)}(q-1)^{2}} .
$$

We show in Section 2 that each maximal subset of pairwise noncommuting elements $N$ of $G=G L(3, q)$ can be modified to contain a given generalized Singer generator or pseudo Singer generator for each maximal torus (see Definition 2.5). This information, together with information about the $p$-singular elements in $N$, leads to our determination of $\omega(G)$. We use the usual notation: for example, $C_{G}(a)$ is the centralizer of an element $a$ in a group $G, N_{G}(H)$ is the normalizer of a subgroup $H$ in $G, G L(n, q)$ is the general linear group of dimension $n$ over a finite field of order $q$, and $S_{n}$ is the symmetric group of degree $n$.

\section{Pairwise noncommuting elements of $G L(3, q)$}

In this section we construct a large subset of pairwise noncommuting elements in $G L(3, q)$. For this purpose we introduce Singer generators and pseudo Singer generator elements.

2.1. An exchange lemma In this subsection we first show a connection between subsets of pairwise noncommuting elements and abelian centralizers, and then determine $\omega(G L(3, q))$ for $q \leq 3$.

LEMMA 2.1 (Exchange lemma). Let $N$ be a set of pairwise noncommuting elements of a group $G$, and let $g \in G$ be such that $C_{G}(g)$ is abelian. Then either $N \cup\{g\}$ is a set of pairwise noncommuting elements, or there is an element $x \in N \cap C_{G}(g)$ such that $(N \backslash\{x\}) \cup\{g\}$ is a set of pairwise noncommuting elements.

Proof. Since $C_{G}(g)$ is abelian, $\left|N \cap C_{G}(g)\right| \leq 1$. If $N \cap C_{G}(g)=\emptyset$ then, for each $x \in N, x g \neq g x$. Thus $N \cup\{g\}$ is a set of pairwise noncommuting elements. So, let $x \in N \cap C_{G}(g)$. We show that $(N \backslash\{x\}) \cup\{g\}$ is a set of pairwise noncommuting 
elements. Suppose that $a, b$ are distinct elements of $(N \backslash\{x\}) \cup\{g\}$ such that $a b=b a$. Since $N \backslash\{x\}$ consists of pairwise noncommuting elements, we can assume that $a \in N \backslash\{x\}$ and $b=g$. It follows that $a \in C_{G}(g)$. Thus $N \cap C_{G}(g)$ contains both $a$ and $x$, which is a contradiction.

We note some simple facts about $\omega(G)$ without proof.

LEMMA 2.2. Let $G$ be a finite group. Then:

(i) for any subgroup $H$ of $G, \omega(H) \leq \omega(G)$;

(ii) for any normal subgroup $N$ of $G, \omega(G / N) \leq \omega(G)$.

Next we compute $\omega(G L(3, q))$ for $q=2,3$.

LEMMA 2.3.

$$
\omega(\operatorname{GL}(3, q))= \begin{cases}57 & \text { if } q=2 \\ 1067 & \text { if } q=3\end{cases}
$$

Proof. We have $G L(3,2) \cong P S L(2,7)$ and, by [1, Lemma 4.4], $\omega(P S L(2,7))=$ 57. Let $G=G L(3,3)$. A computation using $G A P$ [5] shows that the set of orders of elements of $G$ is $\{1,2,3,4,6,8,13,26\}$ and if $A=\left\{C_{G}(g)|g \in G,| C_{G}(g) \mid=\right.$ $12\}, B=\left\{C_{G}(g)|g \in G,| C_{G}(g) \mid=16\right\}, C=\left\{C_{G}(g)|g \in G,| C_{G}(g) \mid=18\right\}$ and $D=\left\{C_{G}(g)|g \in G,| C_{G}(g) \mid=26\right\}$, then $|A|=468,|B|=351,|C|=104$ and $|D|=144$. It follows that there exist elements $a_{i}, b_{j}, c_{k}, d_{l} \in G$ such that $\left|C_{G}\left(a_{i}\right)\right|=12$ for $1 \leq i \leq 468,\left|C_{G}\left(b_{j}\right)\right|=16$ for $1 \leq j \leq 351,\left|C_{G}\left(c_{k}\right)\right|=18$ for $1 \leq k \leq 104$ and $\left|C_{G}\left(d_{l}\right)\right|=26$ for $1 \leq l \leq 144$. Set $X=\left\{a_{i}, b_{j}, c_{k}, d_{l} \mid 1 \leq\right.$ $i \leq 468,1 \leq j \leq 351,1 \leq k \leq 104,1 \leq l \leq 144\}$. Now each subgroup in $A \cup B \cup$ $C \cup D$ is abelian and $G=\bigcup_{x \in X} C_{G}(x)$. We show that $X$ is a subset of pairwise noncommuting elements and $\omega(G)=|X|$. Let $x, y \in X$ and $x \neq y$ such that $x y=y x$. Then $x \in C_{G}(y)$. Since $C_{G}(y)$ is abelian, it follows that $C_{G}(y) \subseteq C_{G}(x)$. Similarly, $C_{G}(x) \subseteq C_{G}(y)$. Hence $C_{G}(x)=C_{G}(y)$, a contradiction. Thus $X$ is a subset of pairwise noncommuting elements and hence $|X| \leq \omega(G)$. On the other hand, suppose $N$ is a set of pairwise noncommuting elements of $G$ of size $\omega(G)$. Then $N \subseteq G=$ $\bigcup_{x \in X} C_{G}(x)$. For each $a \in X, C_{G}(a)$ is abelian, and hence, $\left|N \cap C_{G}(a)\right| \leq 1$. It follows that $\omega(G) \leq|X|$. This completes the proof.

2.2. An audit of the elements of $G L(3, q)$ For larger $q$ we generalize the approach used for the proof when $q=3$. By considering the actions of elements of $G L(3, q)$ on $V=V(3, q)$, we see that there are five conjugacy classes of abelian element centralizers in $G L(3, q)$.

Let $g \in G L(3, q)$ and $V=\bigoplus_{f} V_{f}$ be a primary decomposition of $V$ as $F\langle g\rangle$ module, where the sum is over all monic irreducible polynomials $f \in F[t]$ (see [6, Theorem 7.1 and Lemma 8.10]). Thus each $V_{f}$ is $g$-invariant and if $V_{f} \neq 0$ then the restriction $\left.g\right|_{V_{f}}$ to $V_{f}$ has characteristic polynomial $f^{a_{f}}$ for some $a_{f} \geq 1$. We enumerate the possibilities: 
(i) $g$ is irreducible, $V=V_{f}$, where $\operatorname{deg} f=3, a_{f}=1$.

(ii) $V=V_{f_{1}} \oplus V_{f_{2}}$, where $\operatorname{deg} f_{1}=1, \operatorname{deg} f_{2}=2$ and $a_{f_{1}}=a_{f_{2}}=1$.

(iii) $V=V_{f_{1}} \oplus V_{f_{2}} \oplus V_{f_{3}}$, where $\operatorname{deg} f_{i}=1=a_{f_{i}}$ for $i=1,2$, 3; in this case $q \geq 4$.

(iv) $V=V_{f_{1}} \oplus V_{f_{2}}$, where $\operatorname{deg} f_{i}=1$ for $i=1,2, a_{f_{1}}=1$ and $a_{f_{2}}=2$. Thus $f_{1}(t)=t-\mu$ and $f_{2}(t)=t-\lambda$, where $\lambda \neq \mu$. There are two possible actions of $g$ on $V$, namely $g$ is conjugate to one of the matrices

$$
A_{1}=\left(\begin{array}{ccc}
\lambda & 1 & 0 \\
0 & \lambda & 0 \\
0 & 0 & \mu
\end{array}\right), \quad A_{2}=\left(\begin{array}{ccc}
\lambda & 0 & 0 \\
0 & \lambda & 0 \\
0 & 0 & \mu
\end{array}\right)
$$

where $\lambda \neq \mu, \lambda \neq 0$ and $\mu \neq 0$ :

(a) $g$ is conjugate to $A_{1}$ and $C_{G L(3, q)}(g)$ is abelian of order $q(q-1)^{2}$ consisting of all matrices of the form

$$
A=\left(\begin{array}{ccc}
\alpha & \beta & 0 \\
0 & \alpha & 0 \\
0 & 0 & \gamma
\end{array}\right)
$$

with $\alpha \neq 0$ and $\gamma \neq 0$.

(b) $g$ is conjugate to $A_{2}$ and $C_{G L(3, q)}(g) \cong G L(2, q) \times G L(1, q)$ is nonabelian of order $q\left(q^{2}-1\right)(q-1)^{2}$; moreover, each of these elements $g$ centralizes an element of type (ii).

(v) $\operatorname{deg} f=1, a_{f}=3$ and $f(t)=t-\lambda$, for some $\lambda \neq 0$. There are three possible actions of $g$ on $V$, namely $g$ is conjugate to one of the matrices

$$
B_{1}=\left(\begin{array}{ccc}
\lambda & 1 & 0 \\
0 & \lambda & 1 \\
0 & 0 & \lambda
\end{array}\right), \quad B_{2}=\left(\begin{array}{ccc}
\lambda & 1 & 0 \\
0 & \lambda & 0 \\
0 & 0 & \lambda
\end{array}\right), \quad B_{3}=\left(\begin{array}{ccc}
\lambda & 0 & 0 \\
0 & \lambda & 0 \\
0 & 0 & \lambda
\end{array}\right) \text { : }
$$

(a) $g$ is conjugate to $B_{1}$, and $C_{G L(3, q)}(g)$ is abelian of order $q^{2}(q-1)$ (see Lemma 4.6).

(b) $g$ is conjugate to $B_{2}$, and $C_{G L(3, q)}(g)$ is nonabelian of order $q^{3}(q-1)^{2}$; moreover, each of these elements centralizes an element of type iv(a), for example $B_{2}$ centralizes the matrix $A_{2}$.

(c) $g$ is (conjugate to) $B_{3}$ with nonabelian centralizer $G L(3, q)$; in particular, $g$ centralizes every element of $G L(3, q)$.

LEMMA 2.4. Let $G=G L(3, q)$ and $I=\{\mathrm{i}$, ii, iii, iv(a), v(a) $\}$, and, for $\kappa \in I$, let $S(\kappa)=\left\{C_{G L(3, q)}(g) \mid g\right.$ of type $\left.\kappa\right\}$. Then:

(a) $\quad G=\bigcup_{\kappa \in I}\left(\bigcup_{X \in S(\kappa)} X\right)$;

(b) $\omega(G) \leq \sum_{\kappa \in I}|S(\kappa)|$.

Proof. Part (a) follows from the discussion above. Let $N$ be a maximal subset of pairwise noncommuting elements of $G$, so $\omega(G)=|N|$. Let $X \in \bigcup_{\kappa \in I} S(\kappa)$. Since $X$ is abelian, $|N \cap X| \leq 1$ and hence $\omega(G)=|N| \leq \sum_{\kappa \in I}|S(\kappa)|$. 
2.3. Generalized Singer elements in general linear groups Every element in $G L(3, q)$ has one of the forms as listed in Section 2.2. In this section we introduce Singer generators and pseudo Singer generators, and prove that their centralizers are abelian.

\section{DEFINITION 2.5.}

(a) Let $g \in G L(n, q)$ where $q=p^{k}, p$ is prime, and $|g|=q^{n}-1$. Then $\langle g\rangle$ is called a Singer cycle subgroup of $G L(n, q)$.

(b) Let $V$ be a vector space over a finite field $F$ of dimension 3 and let $\underline{\mathbf{n}}=$ $\left(n_{1}, \ldots, n_{k}\right)$ be $(3),(1,2)$ or $(1,1,1)$. We call $V=V_{n_{1}} \oplus \cdots \oplus V_{n_{k}}$ an $\underline{\mathbf{n}}$-decomposition if, for $i=1,2, \ldots, k, V_{n_{i}}$ is a subspace of $V$ of dimension $n_{i}$.

(c) An element $g$ of $G L(3, q)$ is called an $\underline{\mathbf{n}}$-Singer generator if there is an n-decomposition $V=V_{n_{1}} \oplus \cdots \oplus V_{n_{k}}$ of $V$ such that $g=g_{n_{1}} g_{n_{2}} \cdots g_{n_{k}}$ where, (i) for each $i,\left\langle g_{n_{i}}\right\rangle$ is a Singer cycle subgroup of $G L\left(V_{n_{i}}\right)$, or $\underline{\mathbf{n}}=(1,1,1)$ and $g_{n_{1}}$ has eigenvalue 1 , and (ii) if $n_{i}=n_{j}$ with $i \neq j$, then $c_{g_{n_{i}}}(t) \neq c_{g_{n_{j}}}(t)$, where $c_{g_{n_{i}}}(t)$ is the characteristic polynomial for $g_{n_{i}}$ on $V_{n_{i}}$. We call $\prod_{i=1}^{k}\left\langle g_{n_{i}}\right\rangle$ the n-maximal torus corresponding to $g$.

(d) An element $g$ of $G L(3, q)$ is called a $(1,2)$-pseudo Singer generator if there is a $(1,2)$-decomposition $V=V_{1} \oplus V_{2}$ and distinct primitive elements $\alpha, \beta \in F$ such that $g=g_{1} g_{2}$, where $g_{1} \in G L\left(V_{1}\right)$ acts as $g_{1}: v \mapsto \beta v$ and $g_{2} \in G L\left(V_{2}\right)$ is conjugate to a matrix $\left(\begin{array}{ll}\alpha & 1 \\ 0 & \alpha\end{array}\right)$. We call $\left\langle g_{1}\right\rangle \times C_{G L\left(V_{2}\right)}\left(g_{2}\right)$ the $(1,2)$-maximal pseudo torus corresponding to $g$.

Note that $G L(3, q)$ has no $(1,1,1)$-Singer generator unless $q \geq 4$, and no $(1,2)$ pseudo Singer generator unless $q \geq 3$. Recall the definition of $S(\kappa)$ in Lemma 2.4.

LeMmA 2.6. Let $G=G L(3, q)$, where $q=p^{k} \geq 4$.

(a) Suppose that $g \in G$ is an $\underline{\mathbf{n}}$-Singer generator, where $\underline{\mathbf{n}}=\left(n_{1}, \ldots, n_{k}\right)$ is (3), $(1,2)$ or $(1,1,1)$. Then $\bar{C}_{G}(g)=\prod_{i=1}^{k}\left\langle g_{n_{i}}\right\rangle \in S(\kappa)$ is a subgroup of order $\prod_{i=1}^{k}\left(q^{n_{i}}-1\right)$, for $\kappa=(\mathrm{i})$, (ii) or (iii) respectively. In particular, $p$ does not divide $\left|C_{G}(g)\right|$.

(b) Suppose that $g=g_{1} g_{2} \in G$ is a (1,2)-pseudo Singer generator relative to $V=V_{1} \oplus V_{2}$. Then $C_{G}(g)=\left\langle g_{1}\right\rangle \times B$, where $B=C_{G L\left(V_{2}\right)}\left(g_{2}\right)=Z_{q} \cdot Z_{q-1} \in$ $S(\mathrm{iv}(\mathrm{a}))$ and is conjugate to $\left\{\left(\begin{array}{cc}\alpha & \beta \\ 0 & \alpha\end{array}\right) \mid \alpha, \beta \in F, \alpha \neq 0\right\}$. Moreover, $C_{G}(g)$ has order $q(q-1)^{2}$ and does not contain an $\underline{\mathbf{n}}$-Singer generator for any $\underline{\mathbf{n}}$.

PROOF. (a) Suppose that $V$ is a three-dimensional vector space over a finite field $F$ with size $q$. So by Definition 2.5, we have one of the following:

(1) If $g$ is a (3)-Singer generator of $G$ then $g=g_{3}$. So, by [7, Satz 7.3], $C_{G}(g)=$ $\langle g\rangle \in S(\mathrm{i})$ of order $q^{3}-1$.

(2) If $g$ is a $(1,2)$-Singer generator of $G$ then by Definition 2.5, there is a $g$-invariant $(1,2)$-decomposition $V=V_{1} \oplus V_{2}$ such that $\left.g\right|_{V_{i}}=g_{i}$, for $i=1,2$, and $Z_{q-1} \times$ $Z_{q^{2}-1} \cong\left\langle g_{1}\right\rangle \times\left\langle g_{2}\right\rangle \subseteq C_{G}(g)$. Suppose that $h \in C_{G}(g)$. Now $g$ leaves invariant a unique decomposition $V=V_{1} \oplus V_{2}$ with $\operatorname{dim} V_{1}=1, \operatorname{dim} V_{2}=2$, and moreover, 
$\left(V_{i}^{h}\right)^{g}=\left(V_{i}^{h g h^{-1}}\right)^{h}=\left(V_{i}^{g}\right)^{h}=V_{i}^{h}$, for $i=1,2$, and $V=V_{1}^{h} \oplus V_{2}^{h}$. It follows that, for $i=1,2, V_{i}^{h}=V_{i}$ and hence there exist $h_{1} \in G L\left(V_{1}\right)$ and $h_{2} \in G L\left(V_{2}\right)$ such that $h=h_{1} h_{2}$. Now $g h=h g$ if and only if $g_{i} h_{i}=h_{i} g_{i}$, for $i=1,2$. Therefore $h_{i} \in C_{G L\left(V_{i}\right)}\left(g_{i}\right)$, for $i=1$, 2. By [7, Satz 7.3], $C_{G L\left(V_{i}\right)}\left(g_{i}\right)=\left\langle g_{i}\right\rangle$, for $i=1$, 2. Thus $h \in\left\langle g_{1}\right\rangle \times\left\langle g_{2}\right\rangle$. Hence $C_{G}(g)=\prod_{i=1}^{2}\left\langle g_{i}\right\rangle \in S$ (ii) of order $(q-1)\left(q^{2}-1\right)$.

(3) If $g$ is a $(1,1,1)$-Singer generator of $G$ then, by Definition 2.5, there is a $g$-invariant $(1,1,1)$-decomposition $V=V_{1} \oplus V_{2} \oplus V_{3}$ such that $\left.g\right|_{V_{i}}=g_{i},\left\langle g_{i}\right\rangle=$ $Z_{q-1}$, and the characteristic polynomials of $g_{1}, g_{2}$ and $g_{3}$ are pairwise distinct. So $g$ is conjugate in $G L(3, q)$ to a diagonal matrix with pairwise distinct diagonal entries. It is straightforward to prove that $C_{G}(g)=\prod_{i=1}^{3}\left\langle g_{i}\right\rangle \in S$ (iii) of order $(q-1)^{3}$.

(b) Let $g=g_{1} g_{2}$ and $V=V_{1} \oplus V_{2}$ be as in Definition 2.5(d). Then $C_{G}(g)$ leaves both $V_{1}$ and $V_{2}$ invariant and hence $C_{G}(g)=\left\langle g_{1}\right\rangle \times B \in S($ iv(a)), where $B=$ $C_{G L\left(V_{2}\right)}\left(g_{2}\right)$ and $B$ is conjugate to $\left\{\left(\begin{array}{cc}\alpha & \beta \\ 0 & \alpha\end{array}\right) \mid \alpha, \beta \in F, \alpha \neq 0\right\}$. In particular, $\left|C_{G}(g)\right|=$ $q(q-1)^{2}$ which is not divisible by $q^{3}-1$ or $q^{2}-1$, and so $C_{G}(g)$ does not contain an $\underline{\mathbf{n}}$-Singer generator for $\underline{\mathbf{n}}=(3)$ or $(1,2)$. Also each element of $C_{G}(g)$ has at most two distinct eigenvalues and so $C_{G}(g)$ does not contain a $(1,1,1)$-Singer generator.

LEMMA 2.7. Let $G=G L(3, q)$, where $q=p^{k} \geq 4$. Let $x, y, z$, u be a (3)-Singer generator, (1,2)-Singer generator, $(1,1,1)$-Singer generator and $(1,2)$-pseudo Singer generator of $G$, respectively. Then $\{x, y, z, u\}$ is pairwise noncommuting.

Proof. If $x w=w x$, where $w \in\{y, z, u\}$, then $x \in C_{G}(w)$ and hence $q^{3}-1=|x|$ divides $\left|C_{G}(w)\right|$, which is a contradiction by Lemma 2.6. If $y w=w y$, where $w \in\{z, u\}$ then $y \in C_{G}(w)$ and hence $q^{2}-1=|y|$ divides $\left|C_{G}(w)\right|$, which again contradicts Lemma 2.6. Finally, suppose that $z u=u z$. Then $u \in C_{G}(z)$ with $|u|=$ $p(q-1)$ and $\left|C_{G}(z)\right|=(q-1)^{2}$, again a contradiction.

LEMMA 2.8. Let $N$ be a maximal subset of pairwise noncommuting elements of $G=G L(3, q)$, where $q \geq 4$, let $\underline{\mathbf{n}}=\left(n_{1}, \ldots, n_{k}\right)$ be $(3),(1,2)$ or $(1,1,1)$ and let $g$ be an $\underline{\mathbf{n}}$-Singer generator or $\underline{\mathbf{n}}$-pseudo Singer generator $($ if $\underline{\mathbf{n}}=(1,2)$ ) relative to the $\underline{\mathbf{n}}$-decomposition $V=V_{n_{1}} \oplus \cdots \oplus V_{n_{k}}$, where $\operatorname{dim} V_{n_{i}}=n_{i}$. Then $N$ contains an element $x \in C_{G}(g)$ such that:

(i) $(N \backslash\{x\}) \cup\{g\}$ is also a maximal subset of pairwise noncommuting elements of $G$;

(ii) if $g$ is an $\underline{\mathbf{n}}$-Singer generator then $x$ acts irreducibly on $V_{n_{i}}$ for each $i$;

(iii) if $g$ is $a(1,2)$-pseudo Singer generator then $p$ divides $|x|$.

Proof. By Lemma 2.6, $C_{G}(g)$ is abelian. By Lemma 2.1, the maximality of $N$ implies that there exists $x \in N \cap C_{G}(g)$ such that $N^{\prime}:=(N \backslash\{x\}) \cup\{g\}$ is a maximal subset of pairwise noncommuting elements (possibly $x=g$ ). Suppose first that $g$ is an $\underline{\mathbf{n}}$-Singer generator. We claim that $x$ acts irreducibly on $V_{n_{i}}$ for each $i$. Let $g=g_{1} \cdots g_{k}$ so that $x$ lies in $C_{G}(g)=\prod_{i=1}^{k}\left\langle g_{i}\right\rangle$, say $x=g_{1}^{a_{1}} \cdots g_{k}^{a_{k}}$. Suppose without loss of generality that $g_{1}^{a_{1}}$ acts reducibly on $V_{n_{1}}$. Since, by 
Lemma 2.6, the order of $g_{1}^{a_{1}}$ is not divisible by $p$, it follows from Maschke's theorem that $V_{n_{1}}=U_{1} \oplus \cdots \oplus U_{t}$, where $t \geq 2, U_{i} \neq 0$ and $U_{i}$ is $g_{1}^{a_{1}}$-invariant. Let $\operatorname{dim} U_{i}=m_{i}$. Then there exists an $\left(m_{1}, \ldots, m_{t}, n_{2}, \ldots, n_{k}\right)$-Singer generator $h$ for a maximal torus $T=\left\langle h_{1}\right\rangle \times \cdots \times\left\langle h_{t}\right\rangle \times\left(\prod_{i=2}^{k}\left\langle g_{i}\right\rangle\right)$ containing $x$ relative to the $\left(m_{1}, \ldots, m_{t}, n_{2}, \ldots, n_{k}\right)$-decomposition

$$
V=\left(U_{1} \oplus \cdots \oplus U_{t}\right) \oplus\left(V_{n_{2}} \oplus \cdots \oplus V_{n_{k}}\right) .
$$

Note that $x \in C_{G}(h)=T$ and $T$ is abelian. By Lemma 2.1, there exists $y \in N^{\prime}$ such that $y \in C_{G}(h)$ and $\left(N^{\prime} \backslash\{y\}\right) \cup\{h\}$ is maximal pairwise noncommuting. If $y=g$ then $g_{1}\left(\right.$ of order $q^{n_{1}}-1$ ) lies in $C_{G L\left(V_{n_{1}}\right)}\left(\left.h\right|_{V_{n_{1}}}\right)=\prod_{i=1}^{t}\left\langle h_{i}\right\rangle$, which is a contradiction. Hence $y \in N \backslash\{x\}$ and as $N$ is noncommuting, $y x \neq x y$. However, it follows from the definitions of $h$ and $y$ that both $x, y \in C_{G}(h)$ and $C_{G}(h)$ is abelian. Thus $x y=y x$, which is a contradiction.

Finally let $g$ be a $(1,2)$-pseudo Singer generator, and suppose that $p$ does not divide $|x|$. By Lemma 2.6, it follows that $x=x_{1} x_{2}$ with $x_{1} \in G L\left(V_{1}\right)$ and $x_{2} \in Z\left(G L\left(V_{2}\right)\right)$. Let $y_{2}, y_{2}^{\prime}$ be Singer generators in $G L\left(V_{2}\right)$ such that $\left\langle y_{2}\right\rangle \neq\left\langle y_{2}^{\prime}\right\rangle$, and let $y=x_{1} y_{2}$ and $y^{\prime}=x_{1} y_{2}^{\prime}$. Then $y, y^{\prime} \in C_{G}(x)$ (since $x_{2}$ is central in $G L\left(V_{2}\right)$ ) and $y y^{\prime} \neq y^{\prime} y$ (since $\left\langle y_{2}\right\rangle \neq\left\langle y_{2}^{\prime}\right\rangle$ ). The maximality of $N$ implies that $N \cap C_{G}(x)=\{x\}$ (so that $y, y^{\prime} \notin N$ ). Hence, applying Lemma 2.1 twice, we obtain that $(N \backslash\{x\}) \cup\{y\}$ and $(N \backslash\{x\}) \cup$ $\left\{y^{\prime}\right\}$ are both pairwise noncommuting, and it follows that $(N \backslash\{x\}) \cup\left\{y, y^{\prime}\right\}$ is also pairwise noncommuting, contradicting the maximality of $N$. Thus $p$ divides $|x|$.

LEMMA 2.9. Let $G=G L(3, q)$, where $q=p^{k}>2$, and let $N_{3}$ consist of one (3)Singer generator of $G$ corresponding to each (3)-maximal torus of $G$. Then $\mathrm{N}_{3}$ is a subset of pairwise noncommuting elements of size $|S(\mathrm{i})|=|G| /\left(3\left(q^{3}-1\right)\right)$.

Proof. Let $g, g^{\prime} \in N_{3}$ such that $g g^{\prime}=g^{\prime} g$. By Lemma 2.6, $C_{G}(g)=\langle g\rangle$ and hence $g^{\prime} \in\langle g\rangle$. Similarly, $g \in\left\langle g^{\prime}\right\rangle$. By the definition of $N_{3}, g=g^{\prime}$ and so $N_{3}$ is a subset of pairwise noncommuting elements. By [7, Satz 7.3], $\left|N_{G}(\langle g\rangle)\right|=3|g|=3\left(q^{3}-1\right)$, and hence $\left|N_{3}\right|=\left|G: N_{G}(\langle g\rangle)\right|=|G| /\left(3\left(q^{3}-1\right)\right)$.

LEMMA 2.10. Let $G=G L(3, q)$, where $q=p^{k}>2$. Let $N_{12}$ consist of one $(1,2)$ Singer generator of $G$ corresponding to each $(1,2)$-maximal torus of $G$. Then $N_{12}$ is a subset of pairwise noncommuting elements of size $|S(\mathrm{ii})|=|G| /\left(2\left(q^{2}-1\right)(q-1)\right)$.

PROOF. Let $V$ be a vector space over a finite field $F$ with dimension 3 and $|F|=q$. Let $g$ and $g^{\prime}$ be $(1,2)-$ Singer generators of $G$ such that $g g^{\prime}=g^{\prime} g$. By Definition 2.5, there exist a one-dimensional subspace $V_{1}$ and a two-dimensional subspace $V_{2}$ of $V$ such that $V=V_{1} \oplus V_{2}$, each $V_{i}$ is $g$-invariant, and $g=g_{1} g_{2}$, where, for $i=1,2,\left\langle g_{i}\right\rangle$ is a Singer cycle subgroup of $G L\left(V_{i}\right)$. Similarly, for $g^{\prime}$, there exist a one-dimensional subspace $V_{1}^{\prime}$ and a two-dimensional subspace $V_{2}^{\prime}$ of $V$ such that $V=V_{1}^{\prime} \oplus V_{2}^{\prime}$, each $V_{i}^{\prime}$ is $g^{\prime}$-invariant, and $g^{\prime}=g_{1}^{\prime} g_{2}^{\prime}$, where, for $i=1,2,\left\langle g_{i}^{\prime}\right\rangle$ is a Singer cycle subgroup of $G L\left(V_{i}^{\prime}\right)$. Since $g g^{\prime}=g^{\prime} g, g^{\prime} \in C_{G}(g)$. By Lemma 2.6, $C_{G}(g)$ and $C_{G}\left(g^{\prime}\right)$ are both abelian so $C_{G}(g)=C_{G}\left(g^{\prime}\right)$. It follows that $\left\langle g_{1}\right\rangle \times\left\langle g_{2}\right\rangle=\left\langle g_{1}^{\prime}\right\rangle \times\left\langle g_{2}^{\prime}\right\rangle$ is a 
(1,2)-maximal torus, and $V_{i}=V_{i}^{\prime}$ for $i=1,2$. However, $N_{12}$ contains only one generator of each $(1,2)$-maximal torus of $V$. Hence $g=g^{\prime}$. Thus $N_{12}$ is a subset of pairwise noncommuting elements. The number of one-dimensional subspaces of $V$ not contained in $V_{2}$ is $\left(q^{3}-1\right) /(q-1)-\left(q^{2}-1\right) /(q-1)$ and the number of twodimensional subspaces of $V$ is $\left(q^{3}-1\right) /(q-1)$. Also the number of Singer cycle subgroups of $G L\left(V_{2}\right)$ is $|G L(2, q)| /\left(2\left(q^{2}-1\right)\right)$. Consequently,

$$
\left|N_{12}\right|=\left(\frac{q^{3}-1}{q-1}-\frac{q^{2}-1}{q-1}\right) \times \frac{|G L(2, q)|}{2\left(q^{2}-1\right)} \times \frac{q^{3}-1}{q-1}=\frac{|G|}{2\left(q^{2}-1\right)(q-1)} .
$$

LEMMA 2.11. Let $G=G L(3, q)$, where $q=p^{k} \geq 4$. Let $N_{111}$ consist of one $(1,1,1)$-Singer generator of $G$ corresponding to each $(1,1,1)$-maximal torus of $G$. Then $N_{111}$ is a subset of pairwise noncommuting elements of size $|S(\mathrm{iii})|=$ $|G| /\left(6(q-1)^{3}\right)$.

Proof. Suppose that $g, g^{\prime} \in N_{111}$ such that $g g^{\prime}=g^{\prime} g$. By Definition 2.5, there exist $g_{1}, g_{2}, g_{3}$ of $G$ such that $g=g_{1} g_{2} g_{3}$ where, for $i=1,2,3, g_{i}$ is a generator of a Singer cycle subgroup of $G L\left(V_{i}\right)$ and $V=V_{1} \oplus V_{2} \oplus V_{3}$. Let $t-\lambda_{i}$ be the characteristic polynomial of $g_{i}$, for $i=1,2,3$. By Definition 2.5, $\lambda_{1}, \lambda_{2}, \lambda_{3}$ are pairwise distinct eigenvalues of $g$. Similarly, there exist $g_{1}^{\prime}, g_{2}^{\prime}, g_{3}^{\prime}$ such that $g^{\prime}=$ $g_{1}^{\prime} g_{2}^{\prime} g_{3}^{\prime}$ and $g^{\prime}$ has three distinct eigenvalues $\lambda_{1}^{\prime}, \lambda_{2}^{\prime}, \lambda_{3}^{\prime}$. According to Lemma 2.6, $C_{G}(g)$ and $C_{G}\left(g^{\prime}\right)$ are both abelian, and since $g g^{\prime}=g^{\prime} g$, then $C_{G}(g)=C_{G}\left(g^{\prime}\right)$. So $\prod_{i=1}^{3}\left\langle g_{i}\right\rangle=\prod_{i=1}^{3}\left\langle g_{i}^{\prime}\right\rangle$. By the definition of $N_{111}$ this implies that $g=g^{\prime}$. Hence $N_{111}$ is a subset of pairwise noncommuting elements. Now we determine $\left|N_{111}\right|$, which is the number of decompositions $V_{1} \oplus V_{2} \oplus V_{3}$. We count ordered triples $\left(V_{1}, V_{2}, V_{3}\right)$ of one-dimensional subspaces such that $V=V_{1} \oplus V_{2} \oplus V_{3}$. The number of one-dimensional subspaces $V_{1}$ of $V$ is $\left(q^{3}-1\right) /(q-1)$ and the number of onedimensional subspaces $V_{2}$ of $V$, where $V_{2} \neq V_{1}$, is $\left(\left(q^{3}-1\right) /(q-1)\right)-1$. Also, the number of one-dimensional subspaces $V_{3}$ of $V$ which are not contained in $V_{1} \oplus V_{2}$ is $\left(q^{3}-1\right) /(q-1)-\left(q^{2}-1\right) /(q-1)$. Thus the number of ordered triples

$$
\left(V_{1}, V_{2}, V_{3}\right) \text { is }\left(\frac{q^{3}-1}{q-1}\right) \cdot\left(\frac{q^{3}-1}{q-1}-1\right) \cdot\left(\frac{q^{3}-1}{q-1}-\frac{q^{2}-1}{q-1}\right)=\frac{|G|}{(q-1)^{3}} .
$$

And as each decomposition has been counted 6 times it follows that $\left|N_{111}\right|=$ $|G| /\left(6(q-1)^{3}\right)$.

LEMMA 2.12. Let $G=G L(3, q)$, where $q=p^{k} \geq 4$. Let $N_{12}^{*}$ consist of one $(1,2)$-pseudo Singer generator of $G$ corresponding to each $(1,2)$-maximal pseudo torus of $G$. Then $N_{12}^{*}$ is a subset of pairwise noncommuting elements of size $|S(\operatorname{iv}(\mathrm{a}))|=|G| /\left(q(q-1)^{3}\right) . \quad$ Moreover, $N_{3} \cup N_{12} \cup N_{111} \cup N_{12}^{*}$ is a subset of pairwise noncommuting elements with $N_{3}, N_{12}, N_{111}$ as in Lemmas 2.9, 2.10 and 2.11, respectively. 
ProOf. Let $V$ be a vector space over a finite field $F$ with dimension 3 and $|F|=q$. Let $g$ and $g^{\prime}$ be $(1,2)$-pseudo Singer generators of $G$ such that $g g^{\prime}=g^{\prime} g$. By Definition 2.5, there exist a one-dimensional subspace $V_{1}$ and a two-dimensional subspace $V_{2}$ of $V$ such that $V=V_{1} \oplus V_{2}$ and $g=g_{1} g_{2}$, where $\left\langle g_{1}\right\rangle$ is a Singer cycle subgroup of $G L\left(V_{1}\right)$ and $g_{2}$ is conjugate to the matrix $b=\left(\begin{array}{ll}\alpha & 1 \\ 0 & \alpha\end{array}\right)$, where $\alpha$ is a primitive element of $F$. We may assume that $g_{2}=b$. Similarly, for $g^{\prime}$, there exist a one-dimensional subspace $V_{1}^{\prime}$ and a two-dimensional subspace $V_{2}^{\prime}$ of $V$ such that $V=V_{1}^{\prime} \oplus V_{2}^{\prime}$ and $g^{\prime}=g_{1}^{\prime} g_{2}^{\prime}$, where $\left\langle g_{1}^{\prime}\right\rangle$ is a Singer cycle subgroup of $G L\left(V_{1}^{\prime}\right)$ and $g_{2}^{\prime}$ is conjugate to the matrix $b^{\prime}=\left(\begin{array}{cc}\alpha^{\prime} & 1 \\ 0 & \alpha^{\prime}\end{array}\right)$ with $\alpha^{\prime}$ a primitive element of $F$. Since $g g^{\prime}=g^{\prime} g, g^{\prime} \in C_{G}(g)$. By Lemma 2.6, $C_{G}(g)$ and $C_{G}\left(g^{\prime}\right)$ are both abelian, so $C_{G}(g)=C_{G}\left(g^{\prime}\right)$. Thus $g$ and $g^{\prime}$ determine the same $(1,2)$-maximal pseudo torus. However, $N_{12}^{*}$ contains only one element of each $(1,2)$-maximal pseudo torus of $V$. Hence $g=g^{\prime}$. Thus $N_{12}^{*}$ is a subset of pairwise noncommuting elements. The number of one-dimensional subspaces of $V$ not contained in $V_{2}$ is $\left(q^{3}-1\right) /(q-1)-\left(q^{2}-1\right) /(q-1)$ and the number of two-dimensional subspaces of $V$ is $\left(q^{3}-1\right) /(q-1)$. An easy computation shows that the number of conjugates of $C_{G L\left(V_{2}\right)}\left(g_{2}\right)$ in $G L\left(V_{2}\right)$ is $|G L(2, q)| /\left(q(q-1)^{2}\right)$. Consequently,

$$
|S(\operatorname{iv}(\mathrm{a}))|=\left|N_{12}^{*}\right|=\left(\frac{q^{3}-1}{q-1}-\frac{q^{2}-1}{q-1}\right) \times \frac{|G L(2, q)|}{q(q-1)^{2}} \times \frac{q^{3}-1}{q-1}=\frac{|G|}{q(q-1)^{3}} .
$$

Finally, according to Lemmas 2.7, 2.9, 2.10, 2.11, we have that $N_{3} \cup N_{12} \cup N_{111} \cup$ $N_{12}^{*}$ is a subset of pairwise noncommuting elements.

COROLlary 2.13. Let $G=G L(3, q)$, where $q=p^{k} \geq 4$. Then

$$
\omega(G) \geq|S(\mathrm{i})|+\mid S(\text { ii })|+| S(\text { iii })|+| S(\text { iv(a) }) \mid=q^{6}+q^{5}+2 q^{4}+2 q^{3}+q^{2} .
$$

ProOF. By Lemmas 2.9, 2.10, 2.11 and 2.12, $N_{3} \cup N_{12} \cup N_{111} \cup N_{12}^{*}$ is a subset of pairwise noncommuting elements of size

$$
\begin{aligned}
& |S(\mathrm{i})|+|S(\mathrm{ii})|+|S(\mathrm{iii})|+|S(\mathrm{iv}(\mathrm{a}))| \\
& \quad=\frac{|G|}{3\left(q^{3}-1\right)}+\frac{|G|}{2\left(q^{2}-1\right)(q-1)}+\frac{|G|}{6(q-1)^{3}}+\frac{|G|}{q(q-1)^{3}} \\
& \quad=q^{6}+q^{5}+2 q^{4}+2 q^{3}+q^{2} .
\end{aligned}
$$

\section{Noncommuting subsets of $p$-elements in finite groups}

In this section we prove a general result about subsets of pairwise noncommuting elements consisting of $p$-elements ( $p$ a prime) in arbitrary finite groups. It is used later in the paper. We denote the number of Sylow $p$-subgroups of a finite group $G$ by $v_{p}(G)$.

LEMMA 3.1. Suppose that $G$ is a finite group and $p$ is a prime number dividing $|G|$. Let $P=P_{1}, P_{2}, \ldots, P_{v_{p}(G)}$ be the Sylow $p$-subgroups and for each $i$ choose 
$x_{i} \in G$ such that $P^{x_{i}}=P_{i}$. If $S$ is a subset of pairwise noncommuting elements of $P \backslash \bigcup_{i=2}^{v_{p}(G)} P_{i}$ then $v_{p}(G) \times|S| \leq \omega(G)$.

PROOF. Let $S=\left\{a_{1}, \ldots, a_{k}\right\}$ be a subset of pairwise noncommuting elements of $P \backslash \bigcup_{i=2}^{v_{p}(G)} P_{i}$. For each $a_{i} \in S, P$ is the unique Sylow $p$-subgroup containing $a_{i}$. Then it is easy to see that, for all $i, S^{x_{i}}=\left\{a_{1}^{x_{i}}, \ldots, a_{k}^{x_{i}}\right\}$ is a subset of pairwise noncommuting elements of $P_{i} \backslash\left(P_{1} \cup \cdots \cup P_{i-1} \cup P_{i+1} \cup \cdots \cup P_{v_{p}(G)}\right)$. Set

$$
X=\bigcup_{i=1}^{v_{p}(G)} S^{x_{i}}=\bigcup_{i=1}^{v_{p}(G)}\left\{a_{1}^{x_{i}}, a_{2}^{x_{i}}, \ldots, a_{k}^{x_{i}}\right\} .
$$

We claim that $X$ is a subset of pairwise noncommuting elements of $G$. Suppose to the contrary that $a_{i}^{x_{k}} a_{j}^{x_{l}}=a_{j}^{x_{l}} a_{i}^{x_{k}}$, with $a_{i}^{x_{k}} \neq a_{j}^{x_{l}}$. If $k=l$ this is not possible since $S^{x_{k}}$ is noncommuting. It follows that $\left\langle a_{i}^{x_{k}}, a_{j}^{x_{l}}\right\rangle$ is an abelian $p$-subgroup of $G$, and so there exists a Sylow $p$-subgroup $P^{x_{t}}$ of $G$ such that $\left\langle a_{i}^{x_{k}}, a_{j}^{x_{l}}\right\rangle \subseteq P^{x_{t}}$. By our remark above, $P^{x_{k}}$ is the unique Sylow $p$-subgroup containing $a_{i}^{a_{k}}$, and so $t=k$. Similarly, $t=l$, and this is a contradiction. Therefore $|X|=v_{p}(G) \times|S| \leq \omega(G)$.

COROLlaRY 3.2. Let $G$ be a finite group and let $p$ be a prime number dividing $|G|$. Suppose that if $P_{i}, P_{j}$ are distinct Sylow p-subgroups of $G$, then $P_{i} \cap P_{j}=1$. Then $v_{p}(G) \leq \omega(G)$.

PROOF. By Lemma 3.1, the proof is straightforward.

As an application of Corollary 3.2, we have the following result that was proved by a different method in [3, Theorem 1, p. 294] for symmetric groups $S_{n}$ for arbitrary $n$.

Corollary 3.3. Let $p$ be a prime number. Then $\omega\left(S_{p}\right) \geq(p-2)$ !.

PROOF. Since $v_{p}\left(S_{p}\right)=(p-2)$ ! and any Sylow $p$-subgroup of $S_{p}$ is of size $p$, the assertion follows from Corollary 3.2.

\section{Proof of Theorem 1.1}

In this section we construct a subset of pairwise noncommuting elements of $G L(n, q)$ consisting of unipotent elements. We begin this section with the following definition.

DEFinition 4.1. Let $V$ be a finite-dimensional vector space over $F$. An endomorphism $x$ of $V$ is called semisimple if the minimal polynomial of $x$ has distinct roots, and is called unipotent whenever it is the sum of the identity and a nilpotent endomorphism.

REMARK 4.2. If char $F=p>0$, and $V$ is a finite-dimensional vector space over $F$, then $x \in G L(V)$ is unipotent if and only if $x^{p^{t}}=1$ for some $t \geq 0$. Also $x$ is semisimple if $p$ does not divide the order of $x$. 
Proposition 4.3. Let $x \in G L(V)$.

(a) There exist unique $x_{s}, x_{u} \in G L(V)$ satisfying the conditions $x=x_{s} x_{u}, x_{s}$ is semisimple, $x_{u}$ is unipotent, $x_{s} x_{u}=x_{u} x_{s}$.

(b) $x_{s}, x_{u}$ commute with any endomorphism of $V$ which commutes with $x$.

(c) If $A$ is an $x$-invariant subspace of $V$, then $A$ is invariant under $x_{s}$ and $x_{u}$.

(d) If $x y=y x(y \in G L(V))$, then $(x y)_{s}=x_{s} y_{s},(x y)_{u}=x_{u} y_{u}$.

Proof. See [6, Ch. VI, Lemma B].

We call $x_{s}$ the semisimple part and $x_{u}$ the unipotent part of $x$. Note that if $x$ is both semisimple and unipotent, then $x=1$.

Definition 4.4. Let $G=G L(n, q)$, where $q=p^{k}>2$ and $n \geq 3$. Let $P$ be the subgroup of $G$ of (upper) unitriangular matrices, that is, matrices with 1 on the diagonal and 0 below it. By [7, Satz 7.1], $P$ is a Sylow $p$-subgroup of $G$. Let $F^{*}=\langle\alpha\rangle$, and, for $j=2, \ldots, n-1$, let $i_{j} \in\{1, \ldots, q-1\}$. Set

$$
A_{\left(i_{2}, \ldots, i_{n-1}\right)}=\left(\begin{array}{ccccc}
1 & 1 & 0 & \ldots & 0 \\
0 & 1 & \alpha^{i_{2}} & \ldots & 0 \\
0 & 0 & 1 & \ldots & 0 \\
\vdots & \vdots & \vdots & \vdots & \vdots \\
0 & 0 & \ldots & 1 & \alpha^{i_{n-1}} \\
0 & 0 & \ldots & 0 & 1
\end{array}\right) .
$$

Let $S=\left\{A_{\left(i_{2}, \ldots, i_{n-1}\right)} \mid i_{j} \in\{1, \ldots, q-1\}\right\}$ and $N_{U}=\bigcup_{g \in G} S^{g}$.

We note that, in the case $n=3, S$ is a subset of elements of $G L(3, q)$ of type v(a), as described in Section 2.2.

LEMMA 4.5. Let $G=G L(n, q)$, where $q=p^{k}>2$. Then $N_{U}$ is a subset of pairwise noncommuting unipotent elements of size $|G| /\left(q^{\left(\begin{array}{c}n \\ 2\end{array}\right)}(q-1)^{2}\right)$.

PROOF. Set $B=A_{\left(i_{2}, \ldots, i_{n-1}\right)}-I$, where $I$ is the identity matrix. We shall show that $A_{\left(i_{2}, \ldots, i_{n-1}\right)} \in P \backslash \bigcup_{g} P^{g}$, where $g \in G \backslash N_{G}(P)$. Suppose, for a contradiction, that there exists $g \in G \backslash N_{G}(P)$ such that $A_{\left(i_{2}, \ldots, i_{n-1}\right)} \in P^{g}$. So $g A_{\left(i_{2}, \ldots, i_{n-1}\right)}=C g$, for some $C \in P$. Let

$$
C=\left(\begin{array}{ccccc}
1 & a_{12} & a_{13} & \ldots & a_{1 n} \\
0 & 1 & a_{23} & \ldots & a_{2 n} \\
\vdots & \vdots & \vdots & \vdots & \vdots \\
0 & 0 & 0 & \ldots & a_{n-1 n} \\
0 & 0 & 0 & \ldots & 1
\end{array}\right)
$$

and set $D=C-I$. Thus $g(I+B)=(I+D) g$, and so $g B=D g$. Since the last row of $D$ is zero, the last row of $D g$ is zero, that is, $(D g)_{n i}=0$ for $1 \leq i \leq n$. On the other hand, for $1 \leq k \leq n,(g B)_{n k}=\sum_{j=1}^{n} g_{n j}(B)_{j k}$. It follows that $g_{n 1}=0$ and, for $2 \leq k \leq n-1, g_{n k} \alpha^{i_{k}}=0$. Hence $g_{n k}=0$, for $1 \leq k \leq n-1$. Similarly, 
$g_{i j}=0$ for $j<i$. Thus $g$ is an upper triangular matrix and hence is in $N_{G}(P)$, which is a contradiction. Hence $A_{\left(i_{2}, \ldots, i_{n-1}\right)}$ lies in $P \backslash \bigcup_{g} P^{g}$. Also, it is easy to see that $A_{\left(i_{2}, \ldots, i_{n-1}\right)} \times A_{\left(j_{2}, \ldots, j_{n-1}\right)}=A_{\left(j_{2}, \ldots, j_{n-1}\right)} \times A_{\left(i_{2}, \ldots, i_{n-1}\right)}$ if and only if $i_{k}=j_{k}$ for $k=2, \ldots, n-1$. Therefore $S=\left\{A_{\left(i_{2}, \ldots, i_{n-1}\right)} \mid i_{k} \in\{1, \ldots, q-1\}\right\}$ is a subset of pairwise noncommuting elements of $P \backslash \bigcup_{g} P^{g}$, and $|S|=(q-1)^{n-2}$. Since the number of Sylow $p$-subgroups is

$$
v_{p}(G)=\frac{\left(q^{n}-1\right)\left(q^{n-1}-1\right) \cdots(q-1)}{(q-1)^{n}},
$$

we obtain by Lemma 3.1, a subset of pairwise noncommuting elements of size

$$
v_{p}(G) \cdot|S|=\frac{|G|}{q^{\left(\begin{array}{c}
n \\
2
\end{array}\right)(q-1)^{2}}} .
$$

LEMMA 4.6. Let $G=G L(3, q)$, where $q=p^{k} \geq 4$. If $u \in N_{U}$ then $C_{G}(u)$ is abelian of order $q^{2}(q-1)$ and $\left|N_{U}\right|=|S(\mathrm{v}(\mathrm{a}))|$. Moreover, if $g \in G$ is an $\left(n_{1}, \ldots, n_{k}\right)$ Singer generator, where $\sum n_{i}=3$, and $x$ is a (1,2)-pseudo Singer generator, then $u g \neq g u$ and $u x \neq x u$.

Proof. Let $u$ be as in the statement. So there exists $g \in G$ such that $u \in S^{g}$. Hence there exists $s \in S$ such that $u=s^{g}$. Let

$$
s=\left(\begin{array}{ccc}
1 & 1 & 0 \\
0 & 1 & \alpha^{i} \\
0 & 0 & 1
\end{array}\right)
$$

where $\langle\alpha\rangle=F^{*}$ and $i \in\{1,2, \ldots, q-1\}$. It follows easily that

$$
C_{G}(s)=\left\{\left(\begin{array}{ccc}
a & b & c \\
0 & a & b \alpha^{i} \\
0 & 0 & a
\end{array}\right) \mid a, b, c \in F, a \neq 0\right\} .
$$

It is clear that $C_{G}(s)$ is abelian of order $q^{2}(q-1)$. Since $C_{G}(u)$ is conjugate to $C_{G}(s)$ in $G, C_{G}(u)$ is abelian of order $q^{2}(q-1)$.

Thus each element $u$ of $N_{U}$ is of type v(a), as defined in Section 2.2, and $C_{G}(u)$ is abelian. Then, since $N_{U}$ is pairwise noncommuting, it follows that $\left|N_{U}\right| \leq|S(\mathrm{v}(\mathrm{a}))|$. Conversely, if $X=C_{G}(g) \in S(\mathrm{v}(\mathrm{a}))$, with $g$ of type v(a), then $g=B_{1}^{h}$ for some $h \in G$ and some $\lambda$, with $B_{1}$ as defined in (2.1) in Section 2.2. Now $C_{G}\left(B_{1}\right)=C_{G}\left(A_{(q-1)}\right)$ and hence $X=C_{G}\left(B_{1}\right)^{h}=C_{G}\left(A_{(q-1)}^{h}\right)$ with $A_{(q-1)}^{h} \in N_{U}$. Since $X$ is abelian and $N_{U}$ is noncommuting, distinct subgroups in $S(\mathrm{v}(\mathrm{a}))$ are centralizers of distinct elements of $N_{U}$, and hence $|S(\mathrm{v}(\mathrm{a}))| \leq\left|N_{U}\right|$. It follows that $|S(\mathrm{v}(\mathrm{a}))|=\left|N_{U}\right|$.

Let $g \in G$ be an $\left(n_{1}, \ldots, n_{k}\right)$-Singer generator, where $\sum n_{i}=3$, and suppose $u g=g u$, so $u \in C_{G}(g)$. By Remark 4.2, $p$ divides the order of $u$ and hence divides $\left|C_{G}(g)\right|$, contradicting Lemma 2.6. 
Now, let $x$ be a $(1,2)$-pseudo Singer generator such that $u x=x u$. So $x \in C_{G}(u)$. Since $C_{G}(u)$ is abelian, $C_{G}(u) \subseteq C_{G}(x)$. Similarly, by Lemma 2.6, $C_{G}(x)$ is abelian of order $q(q-1)^{2}$. It follows that $C_{G}(u)=C_{G}(x)$, a contradiction. This completes the proof.

Finally we prove the main theorem.

Proof of Theorem 1.1. Let $N=N_{3} \cup N_{12} \cup N_{111} \cup N_{12}^{*} \cup N_{U}$. If $q \geq 4$ then, by Corollary 2.13 and Lemma 4.6, $N$ is a subset of pairwise noncommuting elements of $G$ and

$$
\begin{aligned}
|N| & =\sum_{\kappa \in I}|S(\kappa)|=q^{6}+q^{5}+2 q^{4}+2 q^{3}+q^{2}+\frac{|G|}{q^{3}(q-1)^{2}} \\
& =q^{6}+q^{5}+3 q^{4}+3 q^{3}+q^{2}-q-1 .
\end{aligned}
$$

Moreover, $\omega(G) \geq|N|=\sum_{\kappa \in I}|S(\kappa)|$. On the other hand, we observed in Lemma 2.4 that $\omega(G) \leq \sum_{\kappa \in I}|S(\kappa)|$. Thus equality holds. If $q=2$ or 3 , the result follows from Lemma 2.3.

\section{Acknowledgements}

The first author wishes to thank the University of Isfahan for financial support, and the School of Mathematics and Statistics at the University of Western Australia during his nine-month sabbatical. The second author was supported by a Federation Fellowship of the Australian Research Council. The authors thank the anonymous referee for comments that helped improve the result from a lower bound to an exact determination.

\section{References}

[1] A. Abdollahi, A. Akbari and H. R. Maimani, 'Non-commuting graph of a group', J. Algbera 298 (2006), 468-492.

[2] E. A. Bertram, 'Some applications of graph theory to finite groups', Discrete Math. 44 (1983), 31-43.

[3] R. Brown, 'Minimal covers of $S_{n}$ by Abelian subgroups and maximal subsets of pairwise noncommuting elements', J. Combin. Theory Ser. A 49(2) (1988), 294-307.

[4] A. Y. M. Chin, 'On non-commuting sets in an extraspecial p-group', J. Group Theory 8 (2005), 189-194.

[5] The GAP Group, GAP-Groups, Algorithms, and Programming, Version 4.4; 2005 (http://www.gap-system.org).

[6] J. E. Humphreys, Linear Algebraic Groups (Springer, New York, 1975).

[7] B. Huppert, Endliche Gruppen, I (Springer, Berlin, 1967).

[8] D. R. Mason, 'On coverings of a finite group by abelian subgroups', Math. Proc. Cambridge Philos. Soc. 83(2) (1978), 205-209.

[9] B. H. Neumann, 'A problem of Paul Erdős on groups', J. Aust. Math. Soc. Ser. A 21 (1976), 467-472.

[10] L. Pyber, 'The number of pairwise non-commuting elements and the index of the centre in a finite group', J. London Math. Soc. 35(2) (1987), 287-295.

[11] J. P. Serre, 'Sur la dimension cohomologique des groups profinis', Topology 3 (1965), 413-420. 
AZIZOLLAH AZAD, Department of Mathematics, University of Isfahan, Isfahan 81746-73441, Iran

e-mail: a-azad@sci.ui.ac.ir, a-azad@araku.ac.ir

CHERYL E. PRAEGER, School of Mathematics and Statistics, The University of Western Australia, Crawley, WA 6009, Australia e-mail: praeger@maths.uwa.edu.au 